\title{
3D IMAGING USING COHERENT SYNCHROTRON RADIATION
}

\author{
Peter Cloetens $^{1}$, Wolfgang Ludwig ${ }^{1}$, Elodie Boller ${ }^{1}$, FrançOise Peyrin $^{1,2}$, \\ MICHEL SCHLENKER ${ }^{3}$ AND JOSÉ BARUCHEL ${ }^{1}$ \\ ${ }^{1}$ European Synchrotron Radiation Facility, B.P. 220, F-38043 Grenoble, France, ${ }^{2}$ CREATIS, INSA Lyon, \\ F-69621 Villeurbanne, France, ${ }^{3}$ CNRS, Lab. Louis Néel, B.P. 166, F-38042 Grenoble, France \\ e-mail: cloetens@esrf.fr \\ (Accepted August 20, 2002)
}

\begin{abstract}
Three dimensional imaging is becoming a standard tool for medical, scientific and industrial applications. The use of modern synchrotron radiation sources for monochromatic beam micro-tomography provides several new features. Along with enhanced signal-to-noise ratio and improved spatial resolution, these include the possibility of quantitative measurements, the easy incorporation of special sample environment devices for in-situ experiments, and a simple implementation of phase imaging. These 3D approaches overcome some of the limitations of $2 \mathrm{D}$ measurements. They require new tools for image analysis.
\end{abstract}

Keywords: computed tomography, phase contrast, synchrotron radiation.

\section{INTRODUCTION}

From their very discovery, X-rays were used to image the bulk of materials which are non transparent for visible light, by taking advantage of their inhomogeneous absorption. A tremendous progress was obtained, twenty-five years ago, when threedimensional (3D) visualisation became available. This computer-assisted tomographic approach provides the 3D information, starting from a large number of two-dimensional (2D) images (radiographs) recorded at various angular positions of the object, through appropriate algorithms and software ("filtered backprojection", described for instance in Herman (1980)). From the reconstructed 3D data, virtual cuts through the object or perspective renderings can be obtained at will.

Micro-tomography, with spatial resolution better than about $20 \mu \mathrm{m}$, emerged in the last fifteen years (Flannery et al., 1987). Several laboratory instruments have been developed (Rüegsegger et al., 1996; Sasov and Van Dyck, 1998), are commercially available, and produce good results. However, the best images, both in terms of signal-to-noise ratio and of spatial resolution, are obtained on instruments using parallel and monochromatic beams from synchrotron radiation facilities. This paper reports on the new imaging possibilities associated with the availability of third generation sources of synchrotron radiation and the associated needs for adapted 3D analysis tools. We briefly present the aspects of synchrotron radiation which are relevant for micro-tomography, and the experimental set-up implemented at beamline ID19 of the European Synchrotron Radiation Facility (ESRF).
3D imaging overcomes some important limitations of 2D measurements: it makes it possible to quantify a micro-structure without an a priori model, and to follow its evolution in a non-destructive way. The main breakthroughs in absorption contrast tomography are the improved spatial resolution, of the order of one micron, and the possibility of reconstructing the spatial distribution of the linear attenuation coefficient, the signature of the sample composition, quantitatively. In many cases, however, conventional absorption imaging fails: the improved sensitivity of phase imaging is then highly beneficial. The spatial coherence of modern synchrotron beams makes a trivial form of phase imaging, based on propagation, possible. The propagation technique can be used either in a qualitative way, mainly useful for edge detection, or in a quantitative way, involving numerical retrieval of the phase from images recorded at different distances. The combination with tomography allows to reconstruct the electron density in the sample with micrometre resolution.

\section{SYNCHROTRON RADIATION AND MICRO-TOMOGRAPHY}

Synchrotron radiation is the electromagnetic radiation produced by ultra-relativistic electrons (energies of several $\mathrm{GeV}$ ) in a storage ring when they are deviated by a magnetic field. The storage ring consists of a succession of bending magnets and straight sections. The magnetic field is uniform in the bending magnets. It oscillates spatially in the insertion devices (wigglers and undulators) which can be installed in the straight sections between bending magnets. The X-ray beam is emitted in a narrow cone, 
tangent to the curved trajectory of the electrons in the storage ring. Beamlines are located all around the storage ring. Synchrotron radiation sources provide beams with very high intensity and a continuous spectrum, spanning the whole range from infrared to $\mathrm{X}$-rays.

The outstanding features of third generation synchrotron radiation facilities, and more particularly the ESRF, in connection with micro-tomographic applications, are:

\section{1. the very high intensity of the X-ray beam}

2. the availability of photons over a very large energy range (energy $E=1-300 \mathrm{keV}$, wavelength $\lambda=$ $\left.4 \times 10^{-3}-1.2 \mathrm{~nm}\right)$

3. the possibility of tailoring the beam to the requirements of a given experiment by choosing the most appropriate insertion device (wiggler or undulator, with a broad range of variations)

4. the small size of the electron beam cross-section $(<100 \mu \mathrm{m})$ and large distance between source and sample. This leads to a very small angular extension of the source as seen from a point in the specimen, hence to a sizable lateral coherence of the X-ray beam.

These features make it possible to perform micro-tomographic experiments that are improved and/or radically new. The emitted synchrotron beam is processed by either a perfect crystal or a multilayer monochromator. This results in a parallel and monochromatic beam providing an exact and quantitative reconstruction, free of beam hardening or geometrical artifacts. The broad energy range available allows to tune the photon energy to a given investigation, generally in order to minimise the deposited energy (dose) and radiation damage in the sample or to maximise the signal-to-noise ratio in the reconstructed volumes. The attenuation by a given chemical element varies abruptly near specific energies corresponding to the electron binding energies. Imaging on both sides of such an absorption edge makes it possible to map the presence of this specific element in the volume. The very high beam intensity reaching the sample allows to improve the spatial resolution down to the micron level, and also to perform 'fast' tomography on dynamic systems. The coherence properties of the beam make it possible to obtain phase images by simply adjusting the sampledetector distance $D$ ("propagation" technique). Two other points are not directly related to the source, but are nevertheless crucial for this type of experiment. 1) The availability of a suitable high resolution CCDbased detector. It consists (Koch et al., 1999) of a scintillator screen converting the $\mathrm{X}$-rays into visible light, magnifying optics and a Peltier cooled CCD. The latter needs to have, simultaneously, a large dynamic range (13 bits in our case), low noise and a short read-out time. We use the Fast REadout LOw Noise (FRELON) camera, developed at the ESRF (Labiche et al., 1996). The effective pixel size of this type of detector can vary between 0.2 and 40 micrometres, the best spatial resolution achieved being $0.5 \mu \mathrm{m} .2$ ) Appropriate image processing software and computing power are required to complete the analysis; they often remain a bottleneck.

Fig. 1 shows the experimental set-up used at the beamline ID19 of the ESRF. The incoming beam can be considered as parallel, the source being situated 150 meters from the sample. In absorption imaging, this monochromatic, lens-less, parallel beam set-up provides an exact and quantitative reconstruction of the linear attenuation coefficient. The main drawback of this approach is that no X-ray magnification is obtained and the spatial resolution mainly results from the resolution of the detector. Unlike visible light or electrons, efficient lenses are extremely difficult to make for hard X-rays, essentially because these interact weakly with matter. Several groups are however making progress toward magnified hard $\mathrm{X}$-ray imaging using diffractive (Di Fabrizio et al., 1999), refractive (Schroer et al., 2002) or reflective (Hignette et al., 2002) optics. It is expected that a spatial resolution of $100 \mathrm{~nm}$ will be achieved in the future. The open geometry of the setup, operating in air, makes it easy to adapt rather sophisticated sample environment devices, such as a specially designed tensile stage (Buffière et al., 1999), a cryostat (Coléou et al., 2001) or a furnace (Bellet et al., submitted). The device should not obstruct the beam when it is rotated over half a turn. This is achieved by setting the sample in a cylindrical enclosure with thin polished Plexiglas (Lucite) or quartz walls that absorb the Xrays only weakly. The total acquisition time is of the order of 5 to 30 minutes for recording 900 projection images, plus around 100 reference images (with beam but without sample) and a dark current image (without X-ray illumination) for flat-field correction. The latter operation consists in dividing, pixel by pixel, the image with sample by a reference image after subtraction of the dark current image in order to correct for inhomogeneities of the incoming beam or the detector. The short acquisition times can be achieved at the highest spatial resolution with the use of a multilayer monochromator (Morawe et al., 1999) giving a bandwidth $\Delta \mathrm{E} / \mathrm{E} \approx 10^{-2}$. The raw data size for one sample typically ranges from 2 Gigabytes $\left(1024^{3}\right.$ voxels) to 16 Gigabytes $\left(2048^{3}\right.$ voxels). Due to the parallel beam geometry, with correct alignment 
of the instrument (assuring perpendicularity between respectively the incoming beam, the rotation axis and the lines of the CCD), the points in a plane perpendicular to the rotation axis are projected for any angular setting on a single line of the CCD. The 3D-reconstruction problem, turning the large set of projections into a volume, is therefore reduced to a sequence of $2 \mathrm{D}$ problems: each slice perpendicular to the rotation axis of the volume is independent of the other slices in the volume. The reconstruction time is kept reasonable $\left(\approx 1\right.$ hour for a $1024^{3}$ volume) by devoting an optimised computer and software to the reconstruction task. However, it remains the lengthiest part of the whole $3 \mathrm{D}$ imaging process.

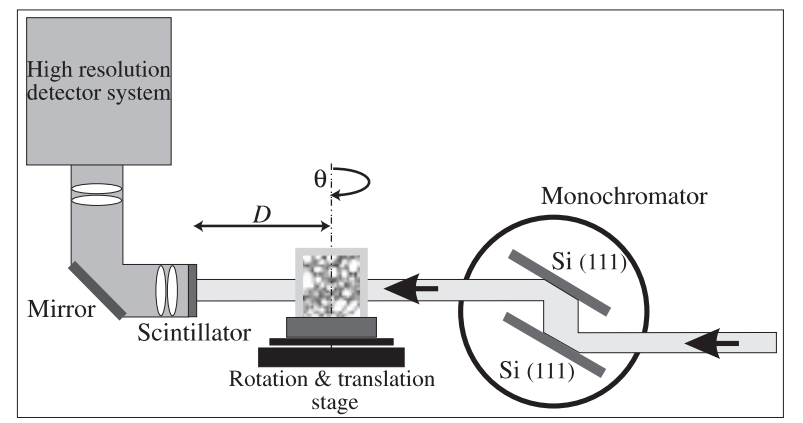

Fig. 1. Principle of parallel and monochromatic beam micro-tomography at beamline IDI9 of the ESRF. The synchrotron beam is monochromatised either by a double silicon crystal monochromator or by an artificial $\mathrm{Al}_{2} \mathrm{O}_{3} / \mathrm{Ru}$ multilayer. Radiographs for a large number of angular settings $\theta$ over a $180^{\circ}$ range are recorded with a CCD-based high resolution detector system. The sample-detector distance $D$ can be varied from $\approx 0$ to $1 \mathrm{~m}$.

\section{D VS. 2D IMAGING}

Most materials studies are based on polished surfaces or thin slices of the material, i.e. on 2D observations. Powerful stereological methods reliably relate the $2 \mathrm{D}$ observations to $3 \mathrm{D}$ statistical information such as the volume, area and length per unit volume of selected features. However, some global quantities cannot be obtained from observations on sections. For instance the determination of the number of objects or contact points requires bulk measurements (Chaix, 2001). Other cases where the $2 \mathrm{D}$ approach is limited are the determination of shapes, studies of connectivity and percolation, ... A restrictive and less than satisfactory solution consists in using a model based on specific assumptions. On the other hand, the threedimensional tomographic data allows to determine the relevant quantities and their distribution directly on an image of the micro-structure. The computational effort is however much heavier and specific algorithms must be implemented. This was for example applied to snow (Coléou et al., 2001) to determine the local curvature of the snow grains and the relationship between the distribution of pores and the curvature. The tomographic volumes are often large enough to be representative of the bulk. They can thus be used as input for numerical calculations in the investigation of the relationship between the micro-structure and function/properties of the material. A compromise must however be made between the field of view (large enough to enclose a representative elementary volume) and the spatial resolution (fine enough to resolve the micro-structure). Limiting factors are the size of the CCD in pixels $N$ (1024 or 2048) and the total number of photons needed to obtain a given signal-to-noise ratio increasing very rapidly with the number of pixels (proportional to $N^{4}$ (Flannery et al., 1987)). The high photon flux and penetrating power of high energy synchrotron beams allows to image larger volumes, but the computational effort for the analysis increases accordingly. In practise most numerical calculations are made on (sub-)volumes with a size in the range $500^{3}-1000^{3}$. Micro-tomography is applied by several groups to bone and bone related diseases (Elliott et al., 1994; Bonse et al., 1994; Rüegsegger et al., 1996; Kinney and Ladd, 1998; Salomé et al., 1999). Tomographic data of trabecular bone obtained with synchrotron radiation can be easily segmented with a simple threshold because the resolution is sufficient compared to the trabecular structure and thanks to the high signal-to-noise ratio in the images. It is possible to perform a much more thorough quantification of the bone architecture than with the classical clinical technique, histomorphometry. The latter approach uses 2D images of thin slices to determine parameters characterising the architecture, such as the volume fraction, the mean thickness of the trabeculae, the average distance between them or the number of interconnections. Actually only two independent values (the surface fraction and the normalised number of intercepts) are measured on the $2 \mathrm{D}$ images, and the architectural parameters are calculated from these two values using a hypothesis: one assumes a bone model consisting of parallel plates or rods (Parfitt et al., 1983). The bulk data, on the other hand, can be used to determine parameters without $a$ priori model. Hildebrand and Rüegsegger (Hildebrand and Rüegsegger, 1997) proposed a definition of a local thickness that can be evaluated at each point of the structure. It is possible to determine the mean value of this quantity, but also its distribution in the volume. The bone type or shape can be considered globally, i.e. it is possible to determine the bone model, or the shape can be characterised locally. The connectivity of the structure, a major topological parameter, is often quantified by the Euler number 
(Serra, 1982; Odgaard and Gundersen, 1993). It can be calculated on discrete three-dimensional data with a method described by Odgaard, the result being usually referred to unit bone volume (Euler density). If there is a singly-connected phase, the Euler density decreases when the connectivity increases. Fig. 2 shows two volume renderings of specimens from the same calcaneum region of different patients. They reveal clear differences in geometry, bone type and topology. The rod-like structure shown in Fig. 2a is much less connected than the plate-like structure in Fig. $2 b$, as confirmed by the Euler densities found, respectively $-2.7 / \mathrm{mm}^{3}$ and $-13.3 / \mathrm{mm}^{3}$.
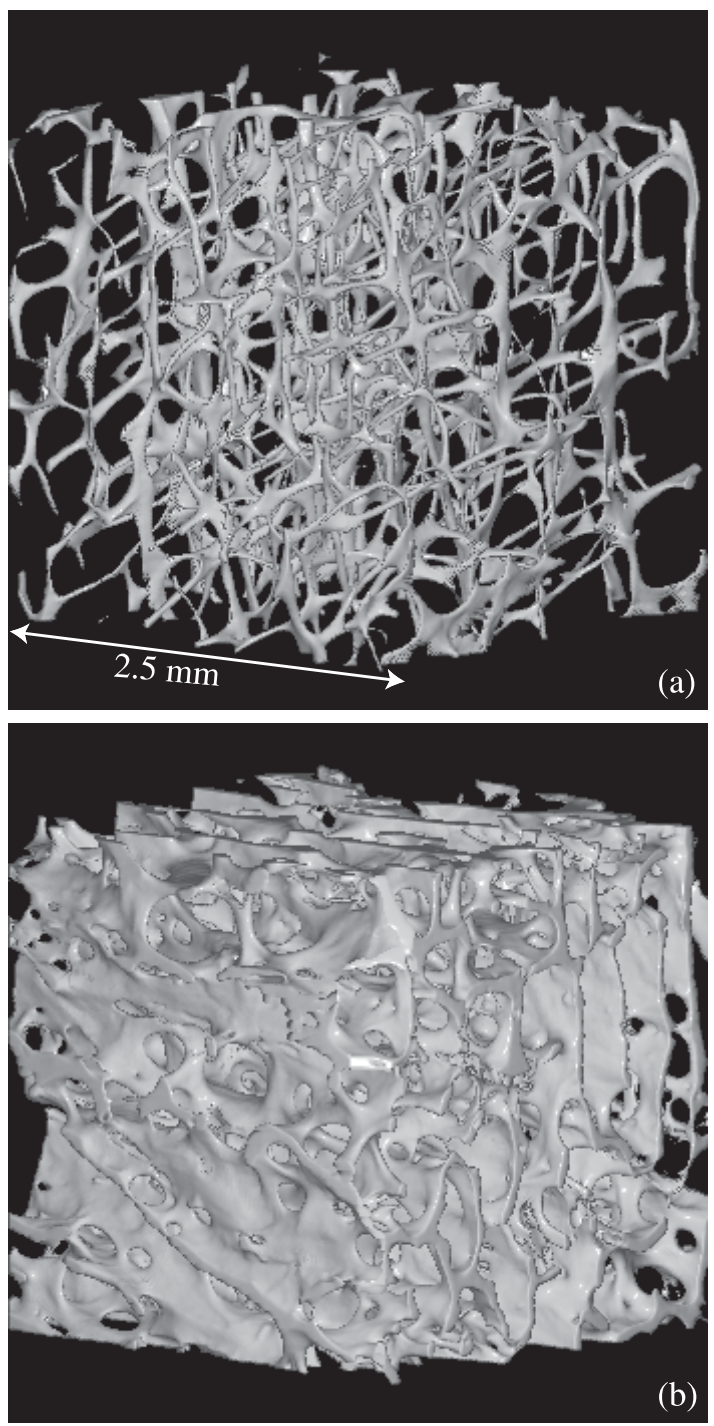

Fig. 2. Volume renderings of bone biopsies from the same calcaneum region of two different patients showing (a) a rod-like structure and (b) a much more connected plate-like structure.

The tomographic data set can be examined with adapted visualisation software, making 3D microscopy possible. Virtual slices in arbitrary directions and the rendering of selected phases in the sample allow to get a better idea of the three-dimensional structure. Local phenomena such as the collapse of foam cells (Elliott et al., 2002), particle-matrix decohesion (Babout et al., 2001), or cracks (Cloetens et al., 1997) can be easily traced in a three-dimensional volume, whereas they will most probably not appear in a single slice. The preparation process for sections or thin slices can introduce artifacts or cover up essential features in the sample (porosity, crack surfaces, ... . ). Because tomography is non-destructive, the original state of the sample is preserved, also after image acquisition. It is thus possible to follow the evolution of the micro-structure and the composition as a function of time, or under the effect of an external load (Buffière et al., 1999) or variations in temperature. This can be done either by sequential tomography, interrupting the process during the time needed for the 3D acquisition, or by fast, real-time tomography, if the acquisition time is small compared to the evolution time of the system. Two-dimensional imaging has the advantage of being faster, most often amenable to real-time visualisation, but it is restricted to surface observations, not necessarily representative of bulk phenomena.

\section{HIGH RESOLUTION AND QUANTITATIVE ABSORPTION TOMOGRAPHY}

The availability of intense, monochromatic Xray beams makes it possible to obtain reconstructed volumes of absorbing specimens with a spatial resolution of the order of one micron. This is at least one order of magnitude better than in the best tomographic devices using laboratory generators. This gain is crucial as the micron scale is often needed to visualise the micro-structure of artificial and natural materials. As already pointed out, the spatial resolution in absorption tomography is mainly determined by the detector. It consists, for high resolution tomography, of a YAG (yttrium aluminium garnet) or LAG (lanthanum aluminium garnet) crystal with a thin scintillating part (5 to $25 \mu \mathrm{m}$ thick doped layer), coupled through a microscope objective to a cooled CCD camera. Improving the spatial resolution while keeping the same signal-to-noise ratio requires the photon flux to be dramatically increased. Simple reasoning suggests that improving the (linear) spatial resolution by a factor of two requires the flux at the sample to be increased by at least a factor of four. Actually, the flux increase needs to be higher, because an improvement in the spatial resolution of the detector results in a decrease of its efficiency. 
As an example where improved spatial resolution is a crucial point, we show the penetration of liquid $\mathrm{Ga}$ in an Al-based alloy. Under specific conditions, a liquid metal can penetrate into the grain boundaries of a polycrystalline solid metal, leading to brittle behaviour of the normally ductile solid. This phenomenon, known as liquid metal embrittlement, was discovered more than a century ago, but the mechanisms leading to rapid penetration along grain boundaries remain poorly understood (Nicholas and Old, 1979; Glickman, 2000). Fig. 3 shows the same virtual slice of an aluminium alloy at various stages. The first tomographic image (Fig. 3a) shows the initial stage of the sample. Fig. $3 b$ was recorded after liquid gallium penetration and after annealing the sample close to room temperature: the white lines that divide the sample into cells indicate the presence of gallium along the grain boundaries. The lines become diffuse and heterogeneous after a second anneal which allows gallium to diffuse into the grains (Fig. 3c): isolated gallium precipitates and cavities can be observed (Ludwig and Bellet, 2000). These experimental observations should corroborate some of the antagonistic models on liquid metal embrittlement proposed in the literature. They also indicate the possibility of using gallium decoration of grain boundaries to obtain 3D visualisation of the grains in a polycrystalline material. An important issue is the correct and automatic segmentation of the 3D data. All voxels belonging to the same grain should be assigned an identical label. This is a non obvious task as the boundary image is weak in some regions due to an unfavourable orientation of the grains. Good results were however obtained with a 3D watershed algorithm (Meyer and Beucher, 1990) implemented by S. Bouchet from Ecole des Mines, Paris. Fig. 4 is a three dimensional rendering of the grains after such a morphologic segmentation. The columnar structure of the extruded material is clearly revealed. An alternative and complementary approach for such investigations was recently developed: X-ray tracking (Poulsen et al., 2001) uses diffraction patterns to determine the shape, position and crystallographic orientation of the grains in bulk polycrystalline samples. It thus provides in the bulk information which electron characterisation methods (Electron Backscattered Diffraction, EBSD) only provide on surfaces. Absorption tomography gives no information on the grain orientation, but it provides better spatial resolution with a shorter acquisition time. It also reveals the morphology of cracks in the material and can be used to study in-situ the interaction of short fatigue cracks with other crystal defects (Ludwig et al., in press).
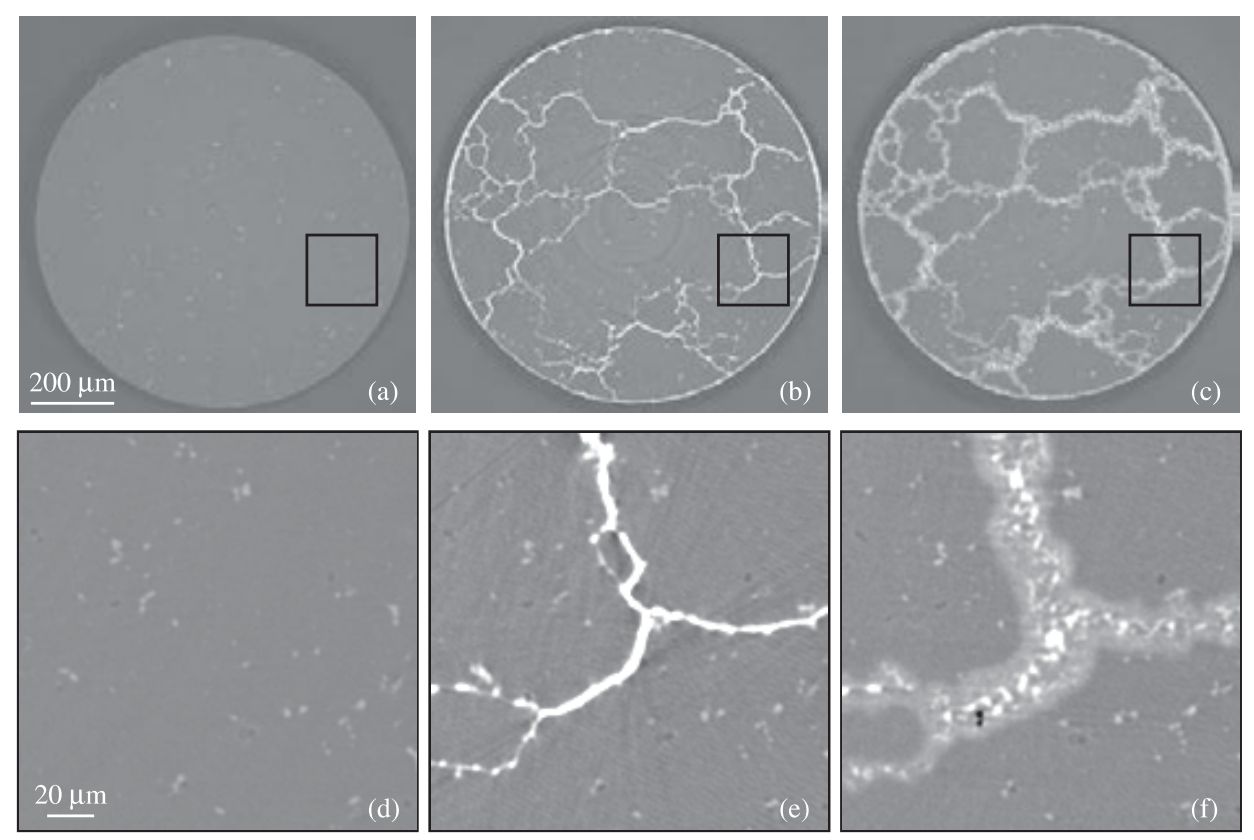

Fig. 3. Virtual slices with a voxel size of $0.9 \mu \mathrm{m}$ of an aluminium alloy (Al 5083) at various stages. (a) Initial stage of the sample. Randomly distributed iron-rich inclusions give rise to the point-like, bright contrasts. (b) Sample after liquid gallium penetration and after annealing the sample for four hours close to room temperature: the white lines that divide the sample into cells indicate the presence of gallium along the grain boundaries. (c) Sample after an additional anneal for 2 hours at $573 \mathrm{~K}$ which allows gallium to diffuse into the grains. The lines become diffuse and heterogeneous, isolated gallium precipitates (white contrast) and cavities (dark contrast) can be observed. 


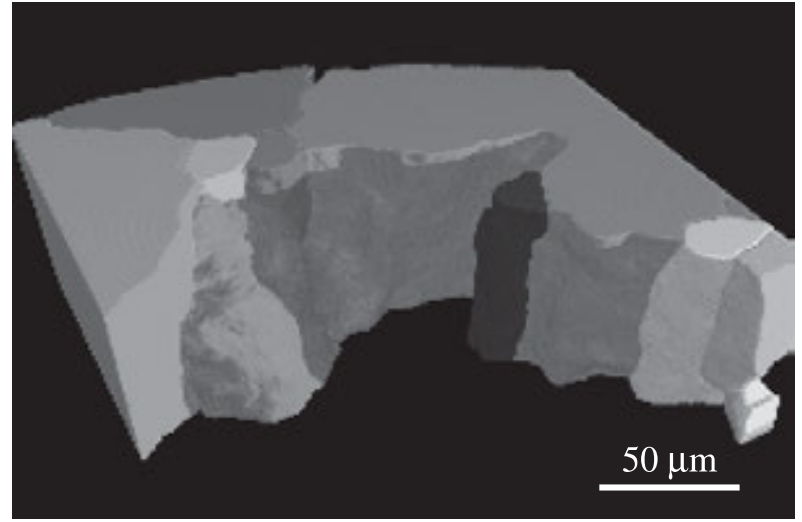

Fig. 4. Three dimensional rendering of the grains in a polycrystalline aluminium alloy after morphological segmentation with a $3 D$ watershed algorithm. The columnar structure of the extruded material can be seen.

A second clear-cut tendency of absorption microtomography using synchrotron radiation is the quantitative evaluation of the images. Quantitative reconstruction, apart from revealing the architecture of the sample, also provides information on its composition. The key point is the monochromaticity of the beam, a basic assumption for tomographic reconstruction. Provided the beam is monochromatic and parallel, the relationship between the intensity $I(x, y)$ transmitted after a path along $z$ in the sample, the incident intensity $I_{o}(x, y)$ and the projection of the linear attenuation coefficient $\mu$, is straightforward and one-to-one:

$$
\int_{\text {path }} \mu(x, y, z) \mathrm{d} z=-\ln \left(\frac{I(x, y)}{I_{o}(x, y)}\right) .
$$

Assuming that this projection is known for a large number of angular orientations of the sample, tomographic reconstruction (or inversion of the Radon transform) makes it possible to quantitatively map the distribution of $\mu(x, y, z)$. This is most often, and in our case always, done with the filtered back-projection algorithm. Alternative methods, e.g. algebraic reconstruction techniques (ART), exist (Herman, 1980; Kak and Slaney, 1988). They are iterative and thus slower, but are useful to reduce the noise by including a priori information or to perform reconstructions even when the data set is incomplete (missing angles, limited number of views, ... ). The linear attenuation coefficient $\mu$ depends, for a given X-ray energy, only on the composition and the density of the material. In the study of bone, Fig. 5 shows that it is now possible not only to access the architecture and porosity of the specimen, but also to measure the mineralisation of the solid parts: it represents a reconstructed slice through a bone biopsy, where different grey levels may be observed within the cortical region (Nuzzo et al., 2001). These grey levels are related to the mineralisation status of the corresponding bone structural unit. This indicates the feasibility of mapping bone mineralisation in 3D, using a suitable model of the bone composition (Nuzzo et al., in press). The agreement between the theoretical and reconstructed linear attenuation coefficients was verified with salt solutions with different concentrations. It can be noted that, when using a laboratory source, one has to accept a polychromatic, divergent beam. The fact that the Xray attenuation of a given element strongly depends on the X-ray energy, together with the divergent beam geometry, shows that laboratory devices can give only qualitative results involving various artifacts.
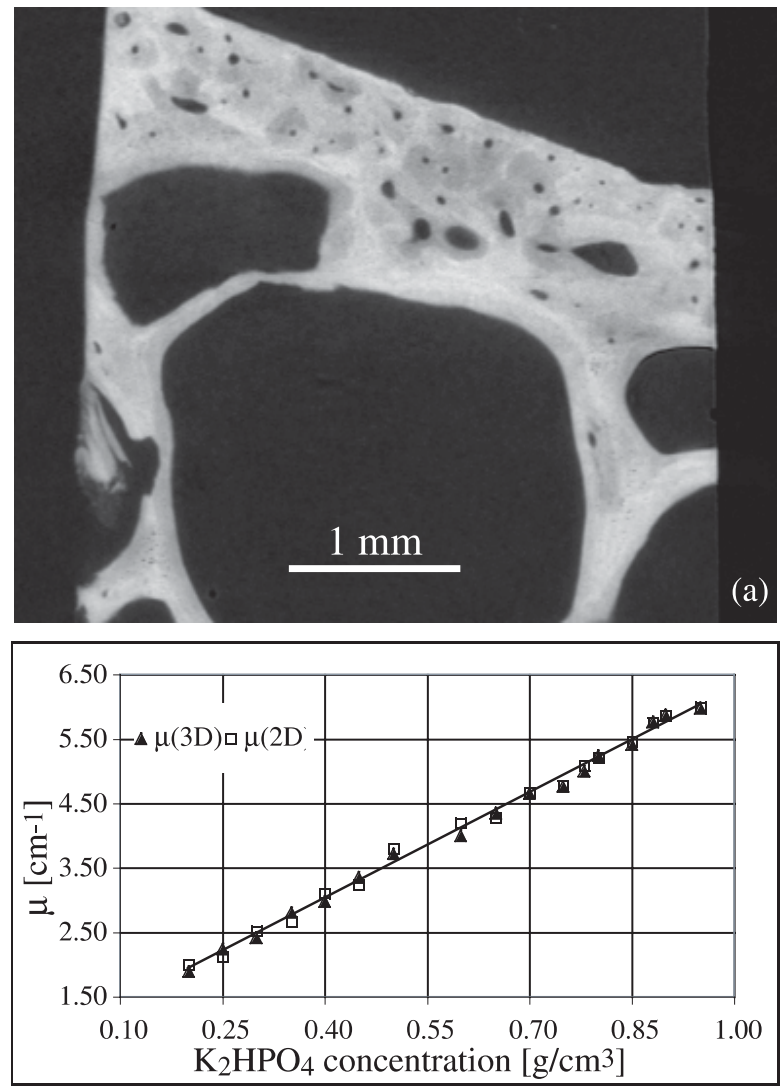

(b)

Fig. 5. (a) Tomographic slice of an iliac crest biopsy sample, showing different grey levels within the cortical bone, related to the mineralisation status of the corresponding bone structural unit (voxel size: $10 \mu \mathrm{m})$. (b) Experimental linear attenuation coefficient of salt solution phantoms as a function of the concentration. Here $\mathrm{K}_{2} \mathrm{HPO}_{4}$ replaces here the insoluble hydroxy-apatite. 


\section{PHASE CONTRAST TOMOGRAPHY}

An X-ray beam passing through a sample undergoes, apart from attenuation, a change in its (optical) phase with respect to what would occur in vacuum. This is formally described by the complex refractive index $n$ of the medium. As it is always very close to unity for hard X-rays, it is usually written as

$$
n=1-\delta+i \beta
$$

The refractive index is defined such that the wavenumber $k=\frac{2 \pi}{\lambda}$ in vacuum and the wavenumber in the material $k^{\prime}$ ar related by $k^{\prime}=n k$. Hence a plane wave propagating in the medium along the $z$-axis is of the form $e^{i n k z}=e^{i(1-\delta) k z} \cdot e^{-\beta k z}$. More generally, the relationship between the wave amplitudes $E(x ; y ; 0)$ right after the specimen and $E_{\text {inc }}(x ; y)$ just before the specimen is given by

$$
E(x, y ; 0)=T(x, y) E_{\text {inc }}(x, y),
$$

with the transmission function $T(x, y)=$ $A(x ; y) \exp [i \varphi(x, y)]$. Its amplitude $A(x ; y)$ corresponds to the attenuation in the material and can be expressed as

$$
A(x, y)=\exp \left(-\frac{2 \pi}{\lambda} \int \beta(x, y, z) \mathrm{d} z\right) .
$$

with $\beta$ linked to the linear attenuation coefficient $\mu=$ $\frac{4 \pi}{\lambda} \beta$. The phase of the transmission function (omitting the phase variation that would occur in vacuum) is given by

$$
\varphi(x, y)=-\frac{2 \pi}{\lambda} \int \delta(x ; y ; z) \mathrm{d} z
$$

$\delta$ is called the refractive index decrement and is, outside the vicinity of the absorption edges, proportional to the electron density $\rho_{e}$, i.e. $\delta=$ $r_{c} \lambda^{2} \rho_{e} /(2 \pi)$ with the classical electron radius $r_{c}=$ $2.810^{-15} \mathrm{~m}$. In the ideally simple case where the incident beam would be a parallel beam and the sample would induce no attenuation of the beam (a pure phase object, $A=1)$, the intensity of the beam right after the specimen $I(x, y ; 0)=|E(x ; y ; 0)|^{2}=I_{\text {inc }}$ would be homogeneous, and a detector placed there would record no phase image. A phase change linear in $x$ acts as a prism and leads to a deviation of the beam proportional to the gradient of the phase. If the phase has non-zero second derivative with respect to $x$, the beam deviation will be inhomogeneous, as it is after a convergent or divergent lens. Hence variations in intensity are expected at non-zero distance from the specimen as a result of interference between parts of the wave that have suffered slightly different deviations. This is indeed true, and the effect, in terms of the wave amplitudes, is simply described by a convolution:

$$
E(x ; y ; D)=P_{D}(x ; y) * E(x ; y ; 0) ;
$$

with the propagator

$$
P_{D}(x, y)=\frac{1}{i \lambda D} \exp \left[i \pi \frac{x^{2}+y^{2}}{\lambda D}\right] .
$$

This effect of simple propagation over a distance $D$ is Fresnel diffraction (Cloetens et al., 1996). It provides contrast from pure phase objects, and, in the case of real samples with both attenuation and phase effects, it combines the two effects. Because the real part $\delta$ of the deviation from unity of the refractive index, while very small $\left(\sim 10^{-6}\right)$, is much larger than its imaginary part $\beta$ describing absorption, phase imaging is much more sensitive to small changes than absorption imaging. For example, the variation in sample thickness that would correspond to $1 \%$ contrast in an aluminiumbased alloy at a photon energy around $25 \mathrm{keV}$ is $20 \mu \mathrm{m}$ for absorption contrast, while it can be only $50 \mathrm{~nm}$ for phase contrast. Fresnel diffraction requires sizable spatial coherence of the X-ray beam. This is available on third-generation synchrotron radiation sources, due to the small cross-sectional area of the electron beam that produces the X-ray beam. In the case of ID19, a long $(150 \mathrm{~m})$ beam-line at ESRF, the coherence width is about $0.1 \mathrm{~mm}$. The striking aspect of this mechanism of phase imaging is its strong dependence on $D$, the specimen-detector distance. At small $D$ ("edge enhancement regime"), thin lines are associated to the jumps in phase due to feature edges, such as the outlines of reinforcing particles in a matrix, or those of the specimen itself. At larger $D$ ("holographic regime"), broad Fresnel fringes cover the image, turning it into a hologram with less and less direct resemblance to the object.

As with attenuation contrast, 2D phase images can be combined into 3D images (volumes) through tomographic reconstruction. This can be done in a qualitative way, mainly useful for edge-detection. Using exactly the same reconstruction procedure as in the case of absorption tomography, one obtains a 3D distribution $o(x, y, z)$. At "small" distance $D$, this distribution is approximately equal to (Cloetens et al., 1997):

$$
\begin{aligned}
o(x ; y ; z) & \approx \mu(x ; y ; z)+ \\
D & \left(\frac{\partial^{2}}{\partial x^{2}}+\frac{\partial^{2}}{\partial y^{2}}+\frac{\partial^{2}}{\partial z^{2}}\right)[1-\delta(x ; y ; z) .
\end{aligned}
$$

The first term is the usual linear attenuation coefficient $\mu(x ; y ; z)$, whereas the second term provides the $3 \mathrm{D}$ 
Laplacian of the real part of the refractive index $n(x, y, z)$ multiplied by the distance $D$. The Laplacian can also be expressed as $\operatorname{div}(\overrightarrow{\mathrm{grad}})$ and a positive value in a given point will tend to deviate the light away from this point, hence can be interpreted as an apparent absorption. The second term will be significant in regions where the density changes abruptly. Phase micro-tomography based on the visualisation of the edges was used, for instance, to understand the mechanisms of degradation in aluminium-SiC composites. It is possible not only to easily visualise the $\mathrm{SiC}$ reinforcing particles, but also to observe the nucleation and propagation of cracks when the material is submitted, in situ, to tensile stress (see Fig. 6). The cracks appear first in the elongated particles, and this imaging technique has shown that the fraction of broken particles is $50 \%$ higher than suggested by surface investigations (Buffière et al., 1999). A noteworthy feature of propagation imaging is that very fine features, such as cracks with submicron opening, normally smaller than the resolution limit of the detector, can be detected in the (2D) radiographs or (3D) tomographs. The reason is that they yield broad, highly visible Fresnel fringes. An important drawback however is the fact that automatic segmentation of edge-enhanced 3D images turns out to be very difficult. Most segmentation algorithms separate regions showing area contrast, i.e. with different grey levels, whereas in this case one should detect the zero crossings (separation between the dark and bright fringes) of the 3D Laplacian. Some work in this field has been done (Vignoles, 2001), but this experimental approach would surely benefit from an adapted, fast and robust segmentation algorithm.
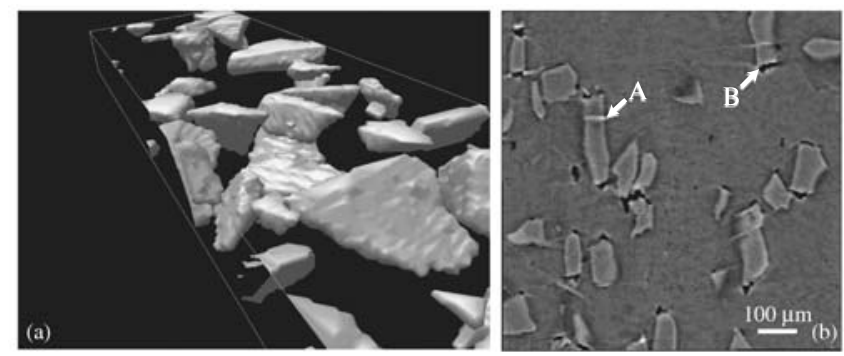

Fig. 6. (a) Volume rendering of SiC particles in an aluminium matrix composite (b) Tomographic slice of a composite after plastic deformation (strain = $510^{-3}$ applied vertically). The $\mathrm{SiC}$ particles are visible through edge enhancement. A crack in one particle is indicated by arrow A. Some matrix particle decohesions are visible in the bulk (arrow B).

Phase imaging based on edge visualisation is not a quantitative technique and its spatial resolution is limited by the width of the fringes used to visualise the borders. A more quantitative version of phase imaging and tomography was developed, involving numerical retrieval of the phase from images recorded at different (typically three or four) distances from the sample (Cloetens et al., 1999). Even in the case of a pure phase object, a single image is generally insufficient because an image at a given distance $D$ is blind to some spatial frequencies in the sample. For example, no contrast is obtained from a periodic phase object with period $a$ at $D$ values that are multiples of $a^{2} / \lambda$ (cf. Talbot effect (Talbot, 1836; Guigay, 1971)). This explains the fact that several images, at different $D$ values, are needed for proper retrieval of the phase map. The intensity distribution $I(x, y ; D)=|E(x, y ; D)|^{2}$ is not directly related to the phase distribution, even with a monochromatic and parallel beam (see Eqs. (3) and (6)). This means that a digital holographic reconstruction procedure has to be used first, to obtain a map of the phase $\varphi(x, y)$ at the exit of the sample. The procedure used at ID19 derives from an approach developed for electron microscopy (Coene et al., 1992; Kirkland, 1984). In it simplest form the holographic reconstruction involves: 1) alignment of the images to the same origin through cross-correlation techniques, 2) filtering of the images, performed in Fourier space and 3) summing the contributions of the different distances. Quantitative phase tomography [holo-tomography (Cloetens et al., 1999)] is obtained by performing a tomographic reconstruction of the phase distributions thus obtained, for many different orientations of the specimen. Holo-tomography is instrumentally very similar to attenuation tomography, but there are two differences. More data must be acquired (about three or four times more, as different values of $D$ are used in the holographic reconstruction process), and the instrument must involve a translation stage. Finally the reconstructed quantity is the spatial distribution of the electron density, therefore to a good approximation the mass density. Holo-tomography was applied to several fields (Cloetens et al., 2001): polymer foams, semi-solid alloys, composite materials, human hair, plant tissue, ... Fig. 7a is a tomographic slice recorded at $D=7 \mathrm{~mm}$, sensitive only to variations in absorption, of an aluminiumsilicon alloy quenched from the semi-solid state. It is impossible to distinguish the two phases, some bright spots appear corresponding to iron-rich inclusions. Fig. $7 \mathrm{~b}$ is a reconstructed map of the variations in refractive index decrement based on four distances, clearly showing the slightly different densities of two (metallurgical) phases $\left(\Delta \rho \approx 0.05 \mathrm{~g} / \mathrm{cm}^{3}\right)$. The grey phase is an aluminium-silicon eutectic (liquid in the semi-solid state) and the dark phase (solid) is essentially pure aluminium with substitutional silicon. Segmentation of the image is in this case much more 
easy and correct. Fig. 7c shows a 3D rendering of the part that was liquid in the semi-solid state, revealing the presence of trapped liquid in the solid at that time. The weak sensitivity of the phase images to smooth variations in the phase (low spatial frequency range) may result however in significant errors and a fluctuating background. As in the case of absorption tomography, the reconstruction is quantitative and can be related to the local composition of the sample. In the example of the semi-solid it allows to estimate the amount of silicon in the eutectic, indicating nonequilibrium solidification due to quenching.

\section{CONCLUSION}

X-ray computed micro-tomography is an invaluable tool to obtain three-dimensional information on a large variety of materials. The use of a third generation synchrotron radiation source opens up new possibilities. The most important features, as far as absorption micro-tomography is concerned, are the very broad choice available in the photon energy, the quantitative evaluation of the experimental data made possible by the beam being monochromatic and parallel, and the improved spatial resolution, now of the order of 1 micron. These techniques are well adapted to in situ experiments, where the material, in an adequate sample environment, is imaged as a function of an external parameter (temperature, stress, ... ). The very small angular size of the source provides, in an instrumentally simple way, phase contrast images with strongly enhanced sensitivity compared to attenuation contrast. Other approaches, using focused beams, and where diffraction or fluorescence are measured, are presently also under development. These 3D observations overcome some of the limitations of 2D measurements. They require new tools for image analysis, involving visualisation, segmentation and parameter extraction.

\section{ACKNOWLEDGMENTS}

The authors are happy to thank their colleagues of the ESRF Topography and Imaging Group, and in particular S. Nuzzo, for their help. We acknowledge D. Fernandez and J.C. Labiche for their contribution to the acquisition setup and A. Hammersley for the implementation of the tomographic reconstruction program.
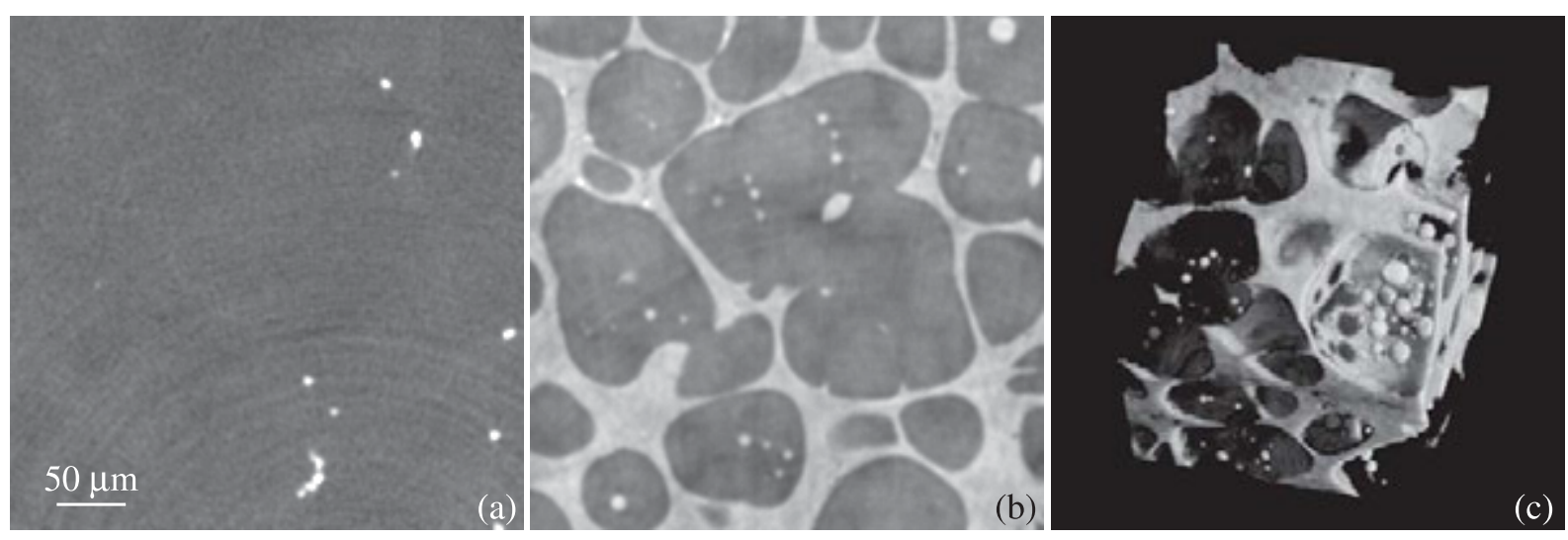

Fig. 7. Tomographic slices of an aluminium-silicon alloy quenched from the semi-solid state, obtained (a) using absorption contrast and (b) using holo-tomography based on four distances. $E=18 \mathrm{keV}, X$-ray wavelength $0.7 \AA$. (c) Volume rendering of the part that was liquid in the semi-solid state, revealing the presence of liquid trapped in the solid part (collaboration with L. Salvo, GPM2, Grenoble).

\section{REFERENCES}

Babout L, Maire E, Buffière JY, Fougères R (2001). Characterization by X-ray computed tomography of decohesion, porosity growth and coalescence in model metal matrix composites. Acta Mater 49:2055-63.

Bellet D, Gorges B, Dallery A, Bernard P, Pereiro E, Baruchel $\mathrm{J}$ (submitted). A $1000^{\circ} \mathrm{C}$ furnace for in-situ X-ray microtomography. J Appl Cryst, submitted.
Bonse U, Busch F, Günnewig O, Beckmann F, Pahl R, Delling G, Hahn M, Graeff W (1994). 3D computed $\mathrm{X}$-ray tomography of human cancellous bone at $8 \mu \mathrm{m}$ spatial resolution and $10^{-4}$ energy resolution. Bone Miner 25:25-38.

Buffière JY, Maire E, Cloetens P, Lormand G, Fougères $R$ (1999). Characterisation of internal damage in a MMCp using X-ray synchrotron phase contrast microtomography. Acta Mater 47:1613-25. 
Chaix JM (2001). Limites de l'information en 2D. In SF2M Journées d'automne 2001, Numéro Hors Série, page 80.

Cloetens P, Barrett R, Baruchel J, Guigay JP, Schlenker M (1996). Phase objects in synchrotron radiation hard Xray imaging. J Phys D-Appl Phys 29:133-46.

Cloetens P, Pateyron-Salomé M, Buffière JY, Peix G, Baruchel J, Peyrin F, Schlenker M (1997). Observation of micro-structure and damage in materials by phase sensitive radiography and tomography. J Appl Phys 81:5878-86.

Cloetens P, Ludwig W, Baruchel J, Van Dyck D, Van Landuyt J, Guigay JP, Schlenker M (1999). Holo-tomography: Quantitative phase tomography with micrometre resolution using hard synchrotron radiation X-rays. Appl Phys Lett 75:2912-4.

Cloetens P, Ludwig W, Helfen L, Salvo L, Mache R, Schlenker M (2001). Quantitative phase contrast tomography using coherent synchrotron radiation. In: Bonse U, ed. Developments in X-Ray Tomography III. Proc SPIE 4503:82-91.

Coene W, Janssen G, Op de Beeck M, Van Dyck D (1992). Phase retrieval through focus variation for ultra-resolution in field-emission transmission electron microscopy. Phys Rev Lett 69:3743-6.

Coléou C, Lesaffre B, Brzoska JB, Ludwig W, Boller E (2001). Three-dimensional snow images by X-ray micro-tomography. Ann Glaciol 32:75-81.

Di Fabrizio E, Romanato F, Gentili M, Cabrini S, Kaulich B, Susini J, Barrett R (1999). High-efficiency multilevel zone plates for keV X-rays. Nature 401:895-8.

Elliott JA, Windle AH, Hobdell J, Eeckhaut G, Olman RJ, Ludwig W, Boller E, Cloetens P, Baruchel J (2002). Insitu deformation of an open-cell flexible polyurethane foam characterised by $3 \mathrm{~d}$ computed microtomography. J Mater Sci 37:1547-55.

Elliott JC, Anderson P, Gao XJ, Wong FS, Davis GR, Dowker SE (1994). Application of scanning microradiography and $\mathrm{X}$-ray micro-tomography to studies of bones and teeth. Journal X-ray Sci Tech 4:102-17.

Flannery BP, Deckman HW, Roberge WG, d'Amico KL (1987). Three dimensional X-ray micro-tomography. Science 237:1439-44.

Glickman, E. E. (2000). Mechanisms of liquid metal embrittlement by simple experiments: from atomistics to life-time. In: Lepinoux J, Maziere D, Pontikis V, Saada G, eds. Multiscal Phenomena in Plasticity, Kluwer Academic Publishers.

Guigay JP (1971). On Fresnel diffraction by onedimensional periodic objects, with application to structure determination of phase objects. Opt Acta 18:677-82.
Herman GT (1980). Image reconstruction from projections. New York: Academic Press.

Hignette O, Rostaing G, Cloetens P, Rommeveaux A, Ludwig W, Freund A (2002). Submicron focusing of hard x-rays with reflecting surfaces at the ESRF. In: McNulty I, ed. X-Ray Micro- and Nano-Focusing: Applications and Techniques II. Proc SPIE 4499:10516.

Hildebrand T, Rüegsegger P (1997). A new method for the model-independent assessment of thickness in threedimensional images. J Microsc 185:67-75.

Kak AC, Slaney M (1988). Principles of Computerized Tomographic Imaging. New York: IEEE Press.

Kinney JH, Ladd AJ (1998). The relationship between threedimensional connectivity and the elastic properties of trabecular bone. J Bone Miner Res 13:839-45.

Kirkland EJ (1984). Improved high resolution image processing in bright field electron micrographs. Ultramicroscopy 15:151-72.

Koch A, Cloetens P, Ludwig W, Labiche JC, Ferrand B (1999). Reading thin-film scintillators with optical microscopes for X-ray imaging. In: Proc. of the 5th Int. Conf. on Inorganic Scintillators and Their Applications SCINT99, Moscow 1999.

Labiche JC, Segura-Puchades J, Van Brussel D, Moy JP (1996). FRELON camera: Fast REadout LOw Noise. ESRF Newsletter 25:41-3.

Ludwig W, Bellet D (2000). Penetration of liquid gallium into the grain boundaries of aluminium: a synchrotron radiation micro-tomographic investigation. Mat Sci Eng A281:198-203.

Ludwig W, Buffière JY, Savelli S, Cloetens P (in press). Study of the interaction of a short fatigue crack with grain boundaries in a cast $\mathrm{Al}$ alloy using $\mathrm{X}$-ray microtomography. Acta Mater, in press.

Morawe C, Pecci P, Peffen JC, Ziegler E (1999). Design and performance of graded multilayers as focusing elements for X-ray optics. Rev Sci Instrum 70:3227-32.

Meyer F, Beucher S (1990). Morphological segmentation. J Visual Commun Image Rep 1:21-46.

Nicholas MG, Old CF (1979). Review liquid metal embrittlement. J Mater Sci 14:1-18.

Nuzzo S, Peyrin F, Martín-Badosa E, Lafage-Proust MH, Boivin G (2001). Assessment of bone mineral content from 3D synchrotron radiation microtomography images. IEEE Trans Nucl Sci 48:859-63.

Nuzzo S, Peyrin F, Cloetens P, Baruchel J, Boivin G (in press). Quantification of the degree of mineralisation of bone in three dimensions using synchrotron radiation micro-tomography. Med Phys, in press. 
Odgaard A, Gundersen HJG (1993). Quantification of connectivity in cancellous bone, with special emphasis on 3-D reconstructions. Bone 14:173-82.

Parfitt AM, Mathews CHE, Villanueva AR, Kleerekoper M (1983). Relationships between surface, volume and thickness of iliac trabecular bone in ageing and in osteoporosis, implications for the micro-anatomic and cellular mechanisms of bone loss. J Clin Invest 72:1396-409.

Poulsen HF, Nielsen SF, Laurdisen EM, Schmidt S, Suter RM, Lienert, U., Margulies, L., Lorentzen, T., and Juul Jensen, D. (2001). Three-dimensional maps of grain boundaries and the stress state of individual grains in polycrystals and powders. J Appl Cryst 34:751-6.

Rüegsegger P, Koller B, Müller R (1996). A microtomographic system for the non destructive evaluation of bone architecture. Calcif Tiss Int 58:24-9.
Salomé M, Peyrin F, Cloetens P, Odet C, Laval-Jeantet AM, Baruchel J, Spanne P (1999). A synchrotron radiation micro-tomography system for the analysis of trabecular bone samples. Med Phys 26:2194-204.

Sasov A, Van Dyck D (1998). Desk-top micro-tomography: gateway to the 3D world. Microscopy and Analysis (European edition), 21-23.

Schroer CG, Meyer J, Kuhlmann M, Benner B, Günzler TF, Lengeler B (2002). Nanotomography based on hard Xray microscopy with refractive lenses. Appl Phys Lett 81:1527-9.

Serra J (1982). Image Analysis and Mathematical Morphology vol.1. London: Academic Press.

Talbot HF (1836). Facts relating to optical science. Phil Mag 9:401-7.

Vignoles GL (2001). Image segmentation for phase-contrast hard X-ray CMT of C/C composites. Carbon 39:167-73. 